\title{
Ab-initio Investigation of the finite-temperatures structural, elastic, and thermodynamic properties of $\mathrm{Ti}_{3} \mathrm{AlC}_{2}$ and $\mathrm{Ti}_{3} \mathrm{SiC}_{2}$
}

\author{
Woongrak Son ${ }^{\mathrm{a}}$, Thien Duong ${ }^{\mathrm{b}}$, Anjana Talapatra $^{\mathrm{b}}$, Huili Gao ${ }^{\mathrm{b}}$, Raymundo Arróyave ${ }^{\mathrm{a}, *}$, Miladin Radovic $^{\mathrm{a}}$ \\ ${ }^{a}$ Department of Materials Science and Engineering, Texas A\&M University, College Station, TX 77843-3123, United States \\ ${ }^{b}$ Department of Mechanical Engineering, Texas A\&M University, College Station, TX 7r843-3123, United States
}

\begin{abstract}
In this work, we calculate the structural, elastic, thermodynamic properties of $\mathrm{Ti}_{3} \mathrm{SiC}_{2}$ and $\mathrm{Ti}_{3} \mathrm{AlC}_{2}$ using a density functional theory (DFT) framework. The vibrational, electronic and quasi-harmonic contributions as well as an anharmonic correction to the total free energy of the system are computed and extrapolated to determine the finite-temperatures properties of the systems. Charge densities, electron localization functions $(\mathrm{ELF})$, the electronic density of states (EDOS) and the vibrational density of states (VDOS) are analyzed in order to deepen our understanding of the interactions giving rise to the calculated properties. Calculated values of Young's modulus, Heat Capacity and Coefficient of Thermal Expansion (CTE) show good agreement with experimental values.
\end{abstract}

Keywords: MAX phases, DFT, elastic constants.

\section{Introduction}

The $\mathrm{M}_{n+1} \mathrm{AX}_{n}$, or MAX phases are ternary carbides or nitrides with a hexagonal crystal structure, wherein the $\mathrm{M}_{n+1} \mathrm{X}_{n}$ layers are interleaved with $\mathrm{A}$ layers. $\mathrm{M}$ is an early transition metal, $\mathrm{A}$ is an A-group element, which is mostly 13 and 14 groups and $\mathrm{X}$ is carbon or nitrogen. MAX phases exhibit a unique combination of properties arising from their atomic bonding and structural characteristics which situates them on the boundary between metals and ceramic bridging the gap between them [1-6]. Due to the metallic nature of the M and A elements, MAX phases tend to be relatively soft and readily machinable (due to weak M-A bonds), with good thermal shock resistance as well as damage tolerance, and in addition are excellent thermal and electrical conductors. Conversely, being carbides and nitrides they exhibit typical ceramic properties and are good refractory materials, are very stable thermodynamically at high temperatures, have good chemical resistance and have relatively low thermal expansion coefficients. These properties make MAX phases amenable for use in the automotive and aerospace industry $[2,3,5]$.

The ' $\mathrm{n}$ ' in the $\mathrm{M}_{n+1} \mathrm{AX}_{n}$ phases, denotes the number of $\mathrm{M}$ layers sandwiched between the A layers.

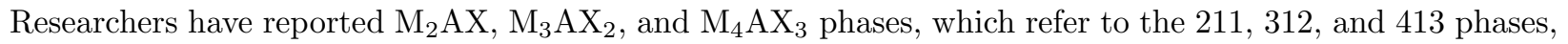
respectively. In 1967, $\mathrm{Ti}_{3} \mathrm{SiC}_{2}$ and $\mathrm{Ti}_{3} \mathrm{GeC}_{2}$ were first synthesized by Jeitschko and Nowotny in powder form [7]; but it wasn't until two decades later that the synthesis of $\mathrm{Ti}_{3} \mathrm{AlC}_{2}$ by Pietzka [8] and that of $\mathrm{Ti}_{3} \mathrm{SiC}_{2}$ in bulk form by Barsoum et al [9] revived interest in this class of materials. In this work, particularly, the 312 MAX phases $\mathrm{Ti}_{3} \mathrm{SiC}_{2}$ and $\mathrm{Ti}_{3} \mathrm{AlC}_{2}$ are investigated. The finite-temperatures structural, elastic, and thermodynamic properties of both phases are calculated.

Lane et al. [10] reported on the crystal structures of the $\mathrm{Ti}_{3} \mathrm{SiC}_{2}$ and $\mathrm{Ti}_{3} \mathrm{GeC}_{2}$ at finite-temperatures. The anisotropical atomic vibration motion of the $\mathrm{Si}$ and $\mathrm{Ge}$ are shown from the neutron diffraction with the highest amplitudes along basal planes. Togo et al. [11] reported on the thermal expansion of the

* Corresponding address: 218 RDMD, Mail Stop 3123, Texas A\&M University, College Station, TX 77843-3123, United States, Tel.: +1 979-845-5416, Fax: +1 979-845-3081

Email address: rarroyave@tamu.edu (Raymundo Arróyave) 
$\mathrm{Ti}_{3} \mathrm{SiC}_{2}$ and $\mathrm{Ti}_{3} \mathrm{AlC}_{2}$. They found that the volume expansion of the $\mathrm{Ti}_{3} \mathrm{SiC}_{2}$ is higher than that of the $\mathrm{Ti}_{3} \mathrm{AlC}_{2}$, and the bulk modulus of $\mathrm{Ti}_{3} \mathrm{SiC}_{2}$ is higher than that of $\mathrm{Ti}_{3} \mathrm{AlC}_{2}$. The inversion in ranking in bulk modulus and thermal expansion is rather unexpected as stiffer lattices tend to have smaller thermal expansion coefficients [12], although Togo et al. did not further discuss these findings.

Knowledge of the thermodynamic properties such as the heat capacity of materials can be used to describe the phase stability in multi phase systems, which are important under the synthesis and processing conditions. Duong et al. $[13,14]$ have calculated the finite-temperatures properties for the $\mathrm{Ti}_{2} \mathrm{AX}(\mathrm{A}=\mathrm{Al}$ or $\mathrm{Ga}$ and $\mathrm{X}=\mathrm{C}$ or $\mathrm{N}$ ) systems. Radovic et al. [15] determined the dependency of elastic properties and damping on temperature for $\mathrm{Ti}_{3} \mathrm{SiC}_{2}, \mathrm{Ti}_{3} \mathrm{GeC}_{2}, \mathrm{Ti}_{3} \mathrm{Si}_{0.5} \mathrm{Al}_{0.5} \mathrm{C}_{2}$ and $\mathrm{Ti}_{2} \mathrm{AlC}$ using resonant ultrasound spectroscopy. They found that the elastic moduli decrease linearly with increasing temperature while the mechanical damping depends weakly on temperature up to $\approx 1273 \mathrm{~K}$ for $\mathrm{Ti}_{3} \mathrm{SiC}_{2}, \mathrm{Ti}_{2} \mathrm{AlC}$ and $\mathrm{Ti}_{3} \mathrm{Si}_{0.5} \mathrm{Al}_{0.5} \mathrm{C}_{2}$, and $\approx$ $700 \mathrm{~K}$ for $\mathrm{Ti}_{3} \mathrm{GeC}_{2}$ after which it increases significantly. Amini et al. [16, 17] used the Vickers hardness and ultrasound experiments to report the mechanical properties of $\mathrm{Ti}_{2} \mathrm{SC}$ and $\mathrm{Cr}_{2} \mathrm{GeC}$. Other than these instances, very little thermodynamic data on MAX phases is available, which exemplifies the need for more such calculations and experiments.

In this work, we calculate the structural, elastic, thermodynamic properties of $\mathrm{Ti}_{3} \mathrm{SiC}_{2}$ and $\mathrm{Ti}_{3} \mathrm{AlC}_{2}$ using a density functional theory (DFT) framework [18]. The vibrational and electronic contributions and anharmonic correction to the total free energy of the system are computed and extrapolated to determine the finite-temperatures properties of the systems. Charge densities, electron localization functions (ELF) ,the electronic density of states (EDOS) and the phonon density of states (PDOS) are analyzed in order to deepen our understanding of the interactions giving rise to the calculated properties.

The organization of this article is as follows: In section 2, we describe the methodology used to calculate the ground state and finite-temperatures properties and the computational parameters used for the same. In section 3 we present the results obtained and analyze the electronic structure and vibrational contributions in the materials under consideration. We also discuss the physical factors underlying the results. Finally, section 4 summarizes the scope of this work and the results obtained.

\section{Methodology}

\subsection{Density Functional Theory (DFT)}

The total energy calculation was performed based on Density Functional Theory (DFT), which is implemented in the Vienna Ab-initio Simulation Package (VASP) [19, 20]. The Local Density Approximation (LDA) and Generalized Gradient Approximation (GGA) were used for the exchange correlation [21, 22]. The Perdew-Burke-Ernzerhof (PBE) is the simplified version of the GGA. The electronic configuration of atoms of titanium, aluminum, silicon and carbon was chosen to be $[\mathrm{Ar}] 3 d^{3} 4 s^{1}$, [Ne] $3 s^{2} 3 p^{1}$, [Ne] $3 s^{2} 3 p^{2}$ and [He] $2 s^{2} 2 p^{2}$ within the projector augmented-wave (PAW) pseudopotentials formalism. Full relaxations were realized by using the Methfessel-Paxton smearing method [23].

\subsection{Elastic properties}

The elastic constants were estimated by stress-strain approach where a set of strains $\left(\varepsilon=\varepsilon_{1}, \varepsilon_{2}, \varepsilon_{3}, \varepsilon_{4}, \varepsilon_{5}\right.$ and $\left.\varepsilon_{6}\right)$ is imposed on a crystal structure. If $\mathrm{A}$ is the lattice vectors specified in Cartesian coordinates, $\varepsilon_{1}, \varepsilon_{2}, \varepsilon_{3}$ and $\varepsilon_{4}, \varepsilon_{5}, \varepsilon_{6}$ are the normal and shear stains, respectively. The deformed lattice vectors are

$$
\bar{A}=A\left|\begin{array}{ccc}
1+\varepsilon_{1} & \frac{\varepsilon_{6}}{2} & \frac{\varepsilon_{5}}{2} \\
\frac{\varepsilon_{6}}{2} & 1+\varepsilon_{2} & \frac{\varepsilon_{4}}{2} \\
\frac{\varepsilon_{5}}{2} & \frac{\varepsilon_{4}}{2} & 1+\varepsilon_{3}
\end{array}\right| .
$$

A set of stresses $\left(\sigma=\sigma_{1}, \sigma_{2}, \sigma_{3}, \sigma_{4}, \sigma_{5}\right.$ and $\left.\sigma_{6}\right)$ for the deformed crystals is generated, which is calculated using density functional theory (DFT) methods. From the $n$ set of strains and the resulting stresses, elastic 
constants are calculated based on Hook's law, as shown below.

$$
\left|\begin{array}{ccc}
C_{11} & \cdots & C_{16} \\
\vdots & & \vdots \\
C_{61} & \cdots & C_{66}
\end{array}\right|=\left|\begin{array}{ccc}
\varepsilon_{1,1} & \cdots & \varepsilon_{1, n} \\
\vdots & & \vdots \\
\varepsilon_{6,1} & \cdots & \varepsilon_{6, n}
\end{array}\right|^{-1}\left|\begin{array}{ccc}
\sigma_{1,1} & \cdots & \sigma_{1, n} \\
\vdots & & \vdots \\
\sigma_{6,1} & \cdots & \sigma_{6, n}
\end{array}\right|
$$

The mechanical stability is determined by the crystal energy, which contains a quadratic form of strain energy:

$$
E=E_{0}+\frac{1}{2} V_{0} \sum_{i, j=1}^{6} C_{i j} \varepsilon_{i} \varepsilon_{j}+O\left(\varepsilon^{3}\right)
$$

By ensuring that the quadratic form of elastic energy is always positive, the mechanical stability criteria of hexagonal phase are given by:

$$
\begin{array}{r}
C_{44}>0, C_{11}>\left|C_{12}\right|, \\
\left(C_{11}+2 C_{12}\right) C_{33}>2 C_{13}^{2} .
\end{array}
$$

\subsection{Finite-temperatures elastic properties}

After calculating the elastic constants on different volume, the finite-temperatures elastic constants can be derived by the dependence of volume on temperature. The latter can be achieved by fitting the free energy equation to the equilibrium volumes at different temperatures. The finite-temperatures elasticity can be derived as follows [24]:

$$
C_{i j}(T)=C_{i j}(V(T))
$$

The calculated elastic constants correspond to the isothermal condition. However, experimentally, the elastic constants are achieved under adiabatic condition. Therefore, we converted the calculated isothermal elastic constants to the adiabatic elastic constants. It can be converted by the Davies's approximation, which relates the isothermal elastic constants to the adiabatic elastic constants by the following equation:

$$
C_{i j}^{S}=C_{i j}^{T}+\frac{T V \lambda_{i} \lambda_{j}}{C_{V}}
$$

,where $C_{i j}^{S}, C_{i j}^{T}$ and $C_{V}$ represents adiabatic elastic constants, isothermal elastic constants and specific heat at constant volume, respectively:

$$
\lambda_{i}=\sum_{j} \alpha_{j} C_{i j}^{T}
$$

Anisotropic coefficients of thermal expansion, $\alpha_{j}$, can be calculated as: $\alpha_{1}=\Lambda_{11}, \alpha_{2}=\Lambda_{22}, \alpha_{3}=\Lambda_{33}$, $\alpha_{4}=\Lambda_{23}, \alpha_{5}=\Lambda_{13}+\Lambda_{13}$ and $\alpha_{6}=\Lambda_{12}+\Lambda_{21}$ :

$$
\Lambda(T)=R^{-1}\left(\frac{\partial R}{\partial V}\right)\left(\frac{\partial V}{\partial T}\right)
$$

$\Lambda(T)$ is an anisotropic tensor of thermal expansion, and $\mathrm{R}$ is a $3 \times 3$ matrix, which combines three lattice verctors: $\mathrm{R}(\mathrm{V})=(\mathrm{a}(\mathrm{V}), \mathrm{b}(\mathrm{V}), \mathrm{c}(\mathrm{V}))^{T}$.

\subsection{Finite-temperature thermodynamics}

The finite-temperatures thermodynamic properties can be derived from the total free energy of a system. In this work, we consider vibrational and electronic contributions and anharmonic correction. The vibrational contribution is obtained by the supercell method, which is implemented in the ATAT package [25]. We calculate force constant tensor of each pair of atoms in the system. We compile all the force constant tensors into the so-called dynamical matrix, which has eigenvalues (frequencies) of the normal modes of oscilation 
in the system. The phonon density of states (PDOS) can be achieved from the frequencies, and PDOS is related to the vibrational free energy through the statistical mechanics [26, 27]:

$$
F_{v i b}(V, T)=\left.k_{B} T \int_{0}^{\infty} \ln \left[2 \sinh \left(\frac{h \nu}{2 k_{B} T}\right)\right] g(\nu)\right|_{V} d \nu
$$

$k_{B}$ is Boltzmann's constant, $\mathrm{h}$ is Planck's constant, $\mathrm{T}$ is temperature, $\mathrm{V}$ is the quasi-harmonic volume, $\nu$ is the frequency and $\left.\mathrm{g}(\nu)\right|_{V}$ is the phonon density of states of the structure corresponding to $\mathrm{V}$.

The electronic degrees of freedom affect to the total free energy of a system. The electronic density of states, $n(\varepsilon)$, and the Fermi function, $\mathrm{f}$, are related to the free energy of electrons by the statistical physics as follows:

$$
\begin{gathered}
F_{e l}(V, T)=E_{e l}(V, T)-T S_{e l}(V, T) \\
E_{e l}(V, T)=\left.\int n(\varepsilon)\right|_{V} f \varepsilon d \varepsilon-\left.\int^{\varepsilon_{F}} n(\varepsilon)\right|_{V} \varepsilon d \varepsilon \\
S_{e l}(V, T)=-\left.k_{B} \int n(\varepsilon)\right|_{V}[f \ln f+(1-f) \ln (1-f)] d \varepsilon
\end{gathered}
$$

Wallace developed the anharmonic free energy equation, which is related to the expansion of the crystal potential.

$$
\begin{gathered}
F_{\text {anhar }}=A_{2} T^{2}+A_{0}+A_{-2} T^{-2}+L \\
A_{2}=\frac{3 k_{B}}{\Theta}(0.0078<\gamma>-0.0154)
\end{gathered}
$$

The Gruneisen parameter, $\gamma$, and the coefficients are based on an empirical data. The anharmonic free energy is only reasonable in the high temperature region since they ignore the last three terms, which cannot be easily determined. Then, Oganov developed the anharmonic free energy, and extended it to all temperature region. Using themodynamic perturbation theory he obtained an expression for the anharmonic free energy as a function of temperature:

$$
\frac{F_{\text {anhar }}}{3 n k_{B}}=\frac{a}{6}\left[\left(\frac{1}{2} \theta+\frac{\theta}{\exp (\theta / T)-1}\right)^{2}+2\left(\frac{\theta}{T}\right)^{2} \frac{\exp (\theta / T)}{(\exp (\theta / T)-1)^{2}} T^{2}\right]
$$

, where a is $1 / 2 A_{2}$ and $\theta$ corresponds to the high temperature Harmonic Debye temperature, defined as $\theta=$ $\frac{\bar{h}}{k_{B}}\left(\frac{5}{3}<\omega^{2}>\right)^{\frac{1}{2}}$.

The total free energy of the system is the summation of the aforementioned energy terms:

$$
F_{\text {total }}(V, T)=E_{0 K}(V)+F_{v i b}(V, T)+F_{e l}(V, T)+F_{\text {anhar }}(V, T)
$$

where $E_{0 K}(V)$ is the zero-temperature energy at each quasi-harmonic volume.

Thermodynamic properties can be calculated using the total free energy:

$$
S=-\frac{\partial F(T)}{\partial T}, C_{P}=T \frac{\partial S}{\partial T}
$$

\section{Results \& Discussion}

\subsection{Ground State Calculation}

The $\mathrm{Ti}_{3} \mathrm{SiC}_{2}$ and $\mathrm{Ti}_{3} \mathrm{AlC}_{2}$ phases form into a nano-layer and hexagonal structure with the space group of $\mathrm{P} 63 / \mathrm{mmc}$, and they are shown in Figure 1. The ground state calculation is optimized by the self-consistent calculation, which is based on density functional theory $[18,28]$. The calculations were performed with LDA and GGA. The results are given in Tabel 1, which agree well with experimented values [29, 30], and other calculated values. We export calculated properties from the Mateirlas Project (www.materialsproject.org) [31], which is open dataset to uncovoer ground state properties of all known inorganic materials. The experimental lattice parameters lie within the LDA-GGA lattice parameters range. The calculated lattice 


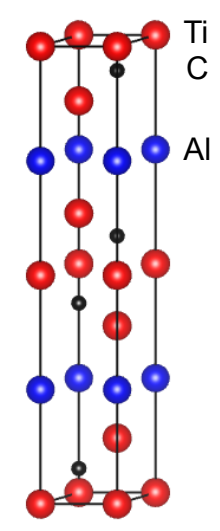

(a) $\mathrm{Ti}_{3} \mathrm{AlC}_{2}$

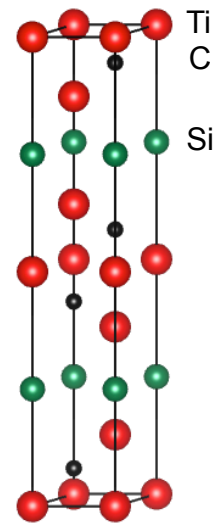

(b) $\mathrm{Ti}_{3} \mathrm{SiC}_{2}$

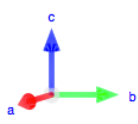

Figure 1: Crystal structure of the (a) $\mathrm{Ti}_{3} \mathrm{AlC}_{2}$ and (b) $\mathrm{Ti}_{3} \mathrm{SiC}_{2}$.

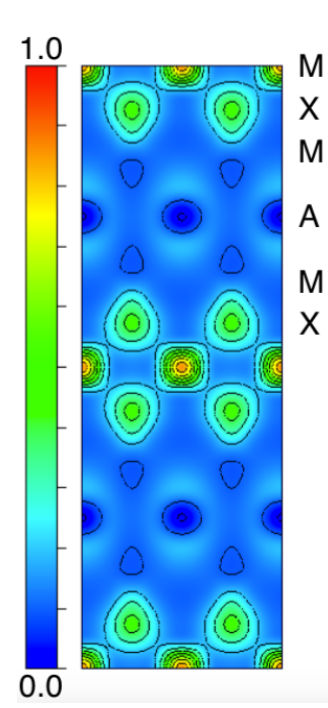

(a) $\mathrm{Ti}_{3} \mathrm{AlC}_{2}$

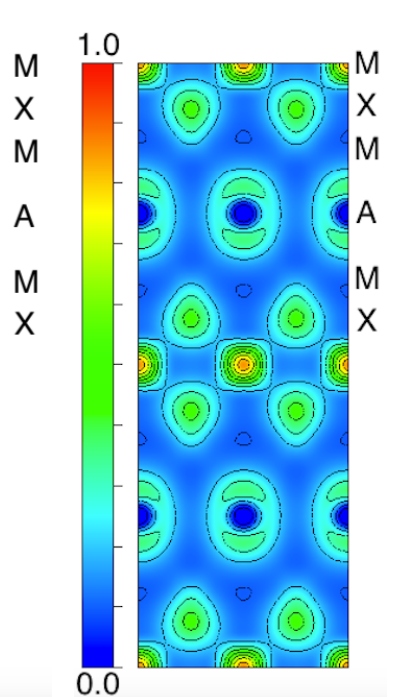

(b) $\mathrm{Ti}_{3} \mathrm{SiC}_{2}$

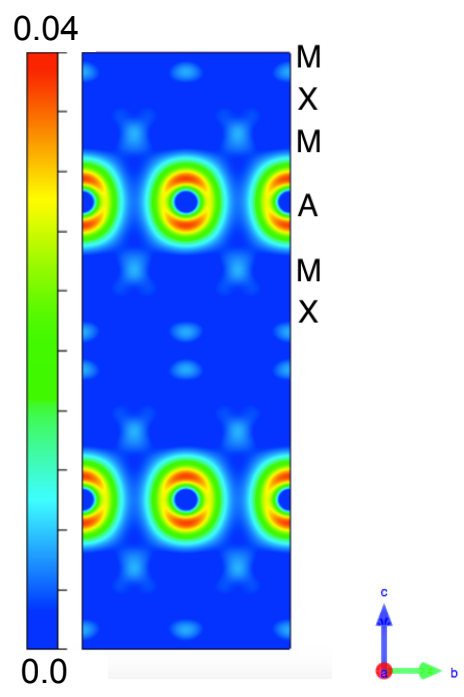

(c) difference

Figure 2: (100) Plane view of Charge Density for the (a) $\mathrm{Ti}_{3} \mathrm{AlC}_{2}$, (b) $\mathrm{Ti}_{3} \mathrm{SiC}_{2}$ and (c) their difference. 


\begin{tabular}{lccccc}
\hline \hline \multirow{2}{*}{ Alloy } & \multirow{2}{*}{ Type } & \multirow{2}{*}{ Potential } & \multicolumn{3}{c}{ Lattice Parameters } \\
\cline { 4 - 6 } & & & $\mathrm{a}(\mathrm{A})$ & $\mathrm{b}(\mathrm{A})$ & $\mathrm{c}(\mathrm{A})$ \\
\hline \multirow{3}{*}{$\mathrm{Ti}_{3} \mathrm{AlC}_{2}$} & Calc. & GGA & 3.083 & 3.083 & 18.652 \\
& Calc. (Ref. 31) & GGA & 3.081 & 3.081 & 18.679 \\
& Exp. (Ref. 27) & & 3.072 & 3.072 & 18.415 \\
\hline \multirow{3}{*}{ Calc. } & GGA & 3.077 & 3.077 & 17.715 \\
$\mathrm{Ti}_{3} \mathrm{SiC}_{2}$ & Calc. (Ref. 31) & GGA & 3.070 & 3.070 & 18.331 \\
& Exp. (Ref. 28) & & 3.065 & 3.028 & 17.453 \\
& & & & 3.065 & 17.756 \\
\hline
\end{tabular}

Table 1: Structural properties of $\mathrm{Ti}_{3} \mathrm{AlC}_{2}$ and $\mathrm{Ti}_{3} \mathrm{SiC}_{2}$

parameters shows that the LDA results underestimate lattice parameter with a discrepancy of $\sim 1.21 \%$, and the GGA results overestimate lattice parameter with a discrepancy of $\sim 0.39 \%$. The GGA results have a smaller discrepancy with experiments and in the remaining of the article, we will present results with GGA calculations. The substitution of $\mathrm{Al}$ with Si mostly affects to the decreasing c lattice parameter value $(5.02 \%)$ than the decreasing a lattice parameter value $(0.19 \%)$. The results suggest that the substitution of aluminum with silicon mostly affects the c lattice parameter. The decreasing lattice parameters of $\mathrm{Ti}_{3} \mathrm{SiC}_{2}$ consistent with the fact that lower atomic volume of the silicon than that of the aluminum.

Fig. 2 shows the (100) plane view of charge density of the $\mathrm{Ti}_{3} \mathrm{AlC}_{2}$ and $\mathrm{Ti}_{3} \mathrm{SiC}_{2}$. The bader analysis, given in Table 2, shows the total charge associated with each atom and charge transfer of each atom. The amount of the charge transfer of each $\mathrm{M}, \mathrm{A}$ and $\mathrm{X}$ elements, is $0.01,0.22$ and 0.07 , respectively, when aluminum is substituted with silicon. The charge density provides a measure of the strength of the ionic bond so that $\mathrm{Ti}_{3} \mathrm{SiC}_{2}$ has stronger ionic bond than $\mathrm{Ti}_{3} \mathrm{AlC}_{2}$. The strong $\mathrm{M}-\mathrm{A}$ bonds in $\mathrm{Ti}_{3} \mathrm{SiC}_{2}$ result to a decreased c lattice parameter value. Fig. 3 shows the (100) plane view of electron localization function (ELF) data of the $\mathrm{Ti}_{3} \mathrm{AlC}_{2}$ and $\mathrm{Ti}_{3} \mathrm{SiC}_{2}$ [32]. ELF value is scaled in the range of 0 to 1 , and provides a (very) qualitative picture of the bonding character within an electronic charge density distribution (in a lattice). The spherical shape of X represents more ionic like bond. The z-Directional localized shape of A represents more covalent like bond. The covalent bond of A elements is localized along the $\mathrm{z}$ direction so that it affects mostly the c lattice parameter.

Fig. 4 shows the electron density of states of the $\mathrm{Ti}_{3} \mathrm{AlC}_{2}$ and $\mathrm{Ti}_{3} \mathrm{SiC}_{2}$. The DOS at the Fermi level mostly dependent on the DOS of titanium, in both of the $\mathrm{Ti}_{3} \mathrm{AlC}_{2}$ and $\mathrm{Ti}_{3} \mathrm{SiC}_{2}$. The calculated DOS reported by Zhou et al. [33] also shows that the DOS at Fermi level mostly dependent on the DOS of titanium, in both of the $\mathrm{Ti}_{3} \mathrm{AlC}_{2}$ and $\mathrm{Ti}_{3} \mathrm{SiC}_{2}$. The Ti-Si bond is stronger than Ti-Al bond, and the titanium of $\mathrm{Ti}_{3} \mathrm{AlC}_{2}$ has more electrons than the titanium of $\mathrm{Ti}_{3} \mathrm{SiC}_{2}$ as shown in bader analysis. It agrees well with the DOS at Fermi level: 2.81 and 2.14 for $\mathrm{Ti}_{3} \mathrm{AlC}_{2}$ and $\mathrm{Ti}_{3} \mathrm{SiC}_{2}$, respectively.

\subsection{Finite Temperature Calculations}

The elastic constants were calculated using stress-strain approach. The elastic moduli of hexagonal structures were estimated based on Voigt's approximation. According to the Voigt's approximation, the bulk, shear and Young's modulus are calculated as follows [36]:

$$
\begin{gathered}
B_{V}=\frac{2\left(C_{11}+C_{12}\right)+4 C_{13}+C_{33}}{9}, \\
G_{V}=\frac{M+12 C_{44}+12 C_{66}}{30},
\end{gathered}
$$




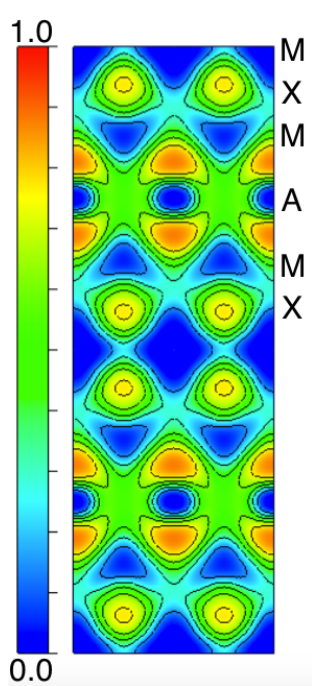

(a) $\mathrm{Ti}_{3} \mathrm{AlC}_{2}$

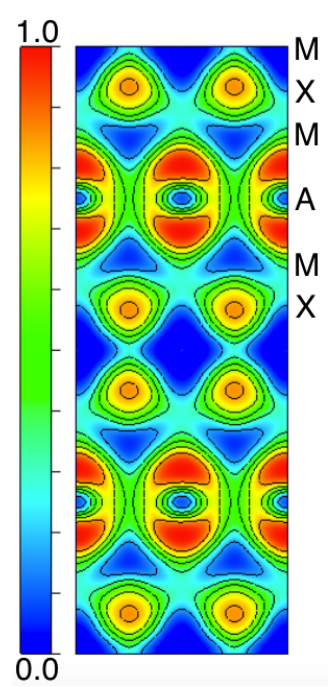

(b) $\mathrm{Ti}_{3} \mathrm{SiC}_{2}$

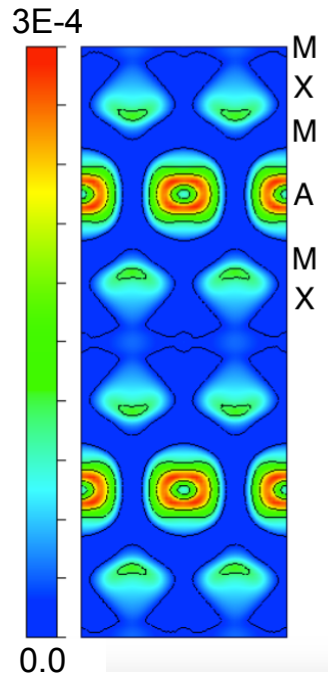

(c) difference

Figure 3: (100) Plane view of Electron Localization Function (ELF) for the (a) $\mathrm{Ti}_{3} A l C_{2}$, (b) $\mathrm{Ti}_{3} \mathrm{SiC}_{2}$ and (c) their difference.

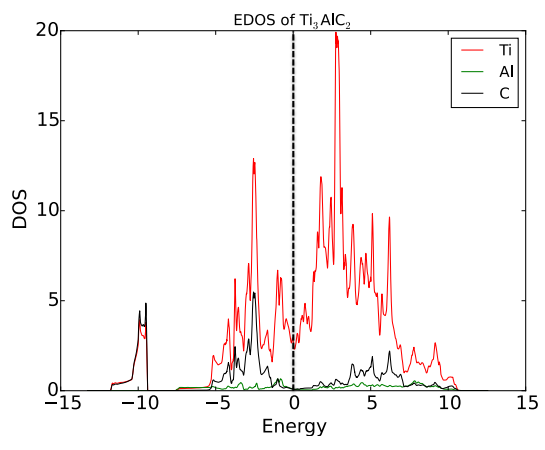

(a) $\mathrm{Ti}_{3} \mathrm{AlC}_{2}$

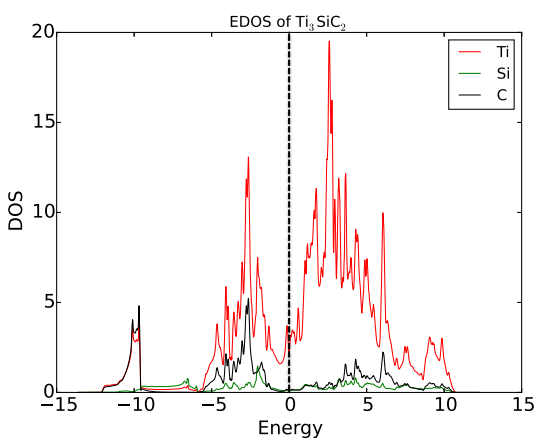

(b) $\mathrm{Ti}_{3} \mathrm{SiC}_{2}$

Figure 4: Electron Density of States of the (a) $\mathrm{Ti}_{3} \mathrm{AlC}_{2}$ and (b) $\mathrm{Ti}_{3} \mathrm{SiC}_{2}$. 


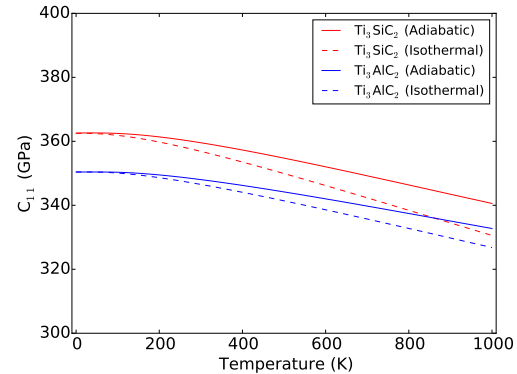

(a) $\mathrm{C}_{11}$

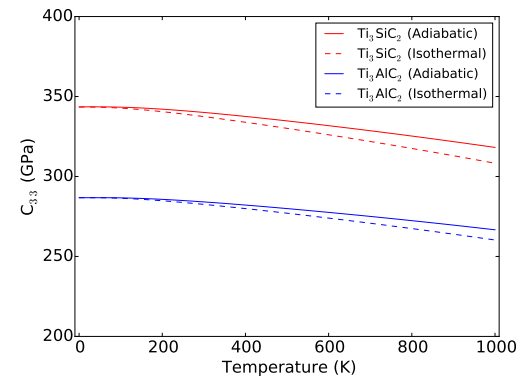

(d) $\mathrm{C}_{33}$

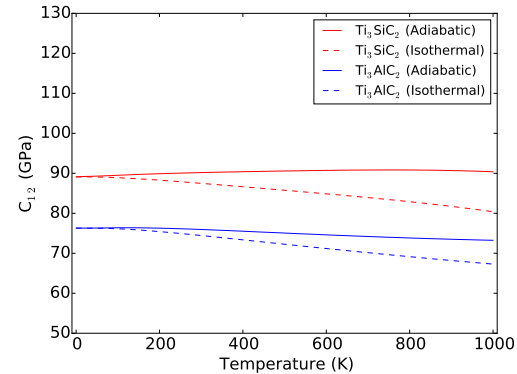

(b) $\mathrm{C}_{12}$

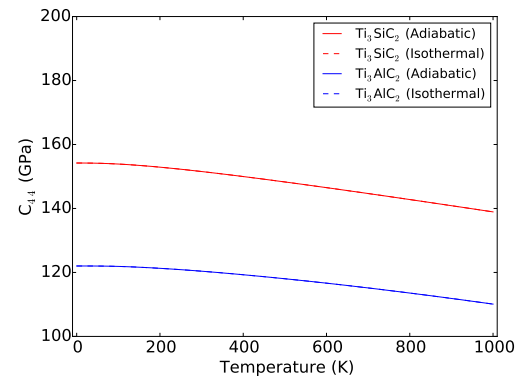

(e) $\mathrm{C}_{44}$

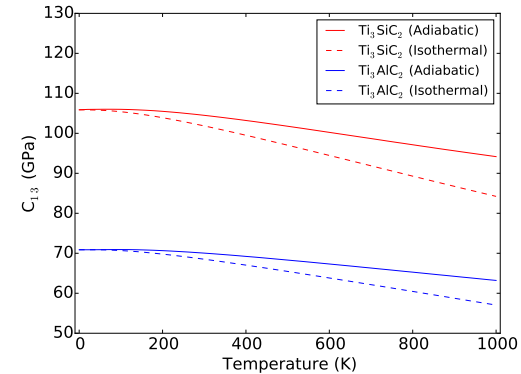

(c) $\mathrm{C}_{13}$

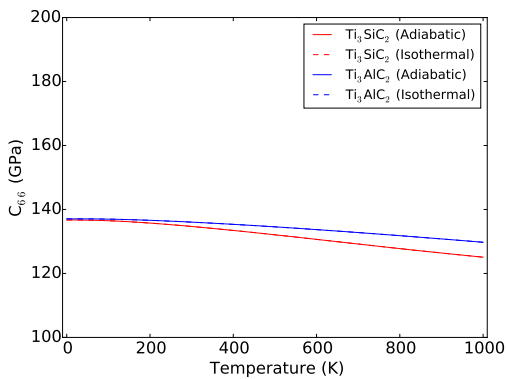

(f) $\mathrm{C}_{66}$

Figure 5: Adiabatic and isothermal elastic constants for $\mathrm{Ti}_{3} \mathrm{AlC}_{2}$ and $\mathrm{Ti}_{3} \mathrm{SiC}_{2}$.

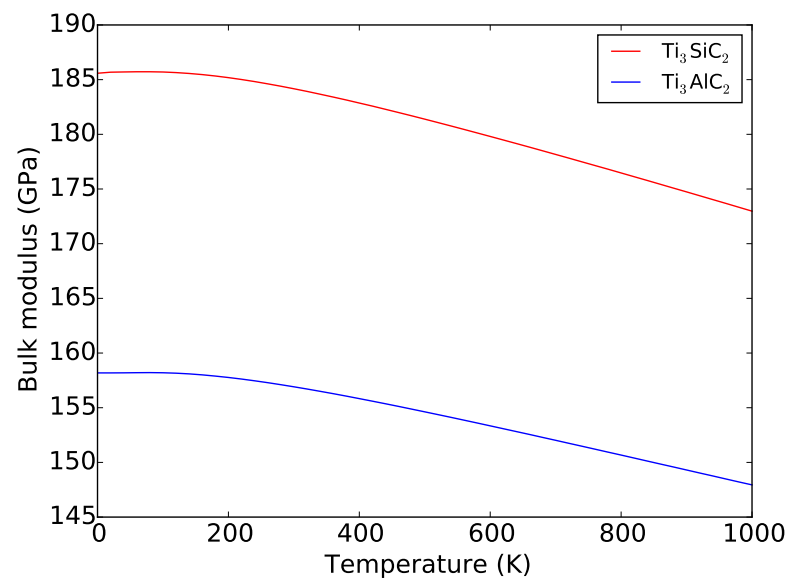

(a) Bulk modulus

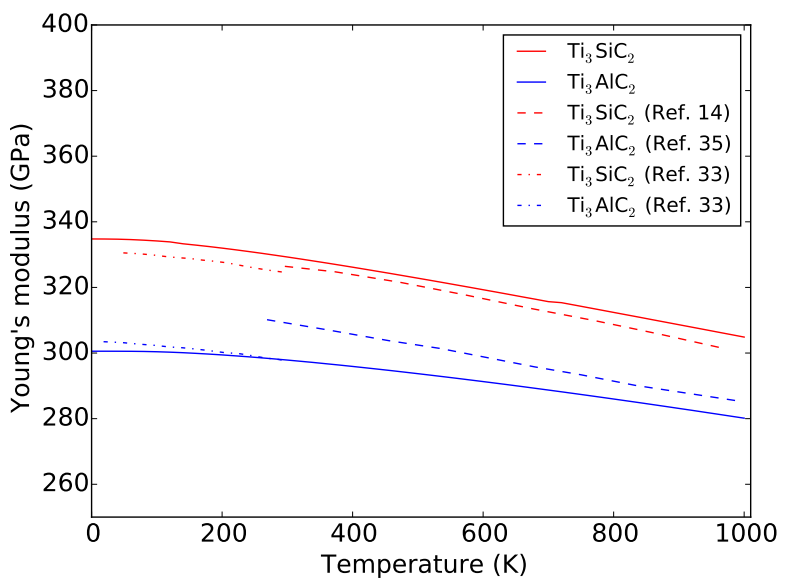

(b) Young's modulus

Figure 6: (a) The Bulk and (b) Young's modulus of $\mathrm{Ti}_{3} \mathrm{AlC}_{2}$ and $\mathrm{Ti}_{3} \mathrm{SiC}_{2}$ as a function of temperature. Both of the Bulk and Young's modulus are calculated using adiabatic elastic constants. The calculated Young's modulus is compared to the experimental value of Young's modulus reported by Radovic et al. [15], Finkel et al. [34] and Wang et al [35]. 


\begin{tabular}{rccc}
\hline \hline & $\mathrm{Ti}(\mathrm{d} 3 \mathrm{~s} 1)$ & $\mathrm{C}(\mathrm{s} 2 \mathrm{p} 2)$ & $\mathrm{Al}(\mathrm{s} 2 \mathrm{p} 1), \mathrm{Si}(\mathrm{s} 2 \mathrm{p} 2)$ \\
\hline $\mathrm{Ti}_{3} \mathrm{AlC}_{2}$ & $1.905(-2.095)$ & $6.565(+2.565)$ & $4.155(+1.155)$ \\
$\mathrm{Ti}_{3} \mathrm{SiC}_{2}$ & $1.883(-2.117)$ & $6.488(+2.488)$ & $5.375(+1.375)$ \\
\hline \hline & $\mathrm{Ti} \mathrm{(total)}$ & $\mathrm{Al} \& \mathrm{Si}($ total$)$ & $\mathrm{C}($ total $)$ \\
\hline $\mathrm{Ti}_{3} \mathrm{AlC}_{2}$ & 11.43 & 26.26 & 8.31 \\
$\mathrm{Ti}_{3} \mathrm{AlC}_{2}$ & 11.3 & 25.95 & 10.75 \\
\hline \hline & $\% \mathrm{Ti}$ & $\% \mathrm{C}$ & $\% \mathrm{Al}, \mathrm{Si}$ \\
\hline $\mathrm{Ti}_{3} \mathrm{AlC}_{2}$ & 24.85 & 57.01 & 18.14 \\
$\mathrm{Ti}_{3} \mathrm{SiC}_{2}$ & 23.50 & 54.10 & 22.40 \\
\hline \hline
\end{tabular}

Table 2: Calculated total charge (charge transfer) of $\mathrm{Ti}_{3} \mathrm{AlC}_{2}$ and $\mathrm{Ti}_{3} \mathrm{SiC}_{2}$ obtained from Bader analysis.

\begin{tabular}{cccccccccc}
\hline \hline Phase & $C_{11}$ & $C_{33}$ & $C_{44}$ & $C_{12}$ & $C_{13}$ & $\mathrm{~B}$ & $\mathrm{G}$ & $\mathrm{E}$ & \\
\hline $\mathrm{Ti}_{3} \mathrm{AlC}_{2}$ & 355.45 & 292.89 & 119.03 & 84.63 & 76.03 & 163.35 & 125.47 & 299.68 & \\
& 355.15 & 296.11 & 114.5 & 74.71 & 69.1 & 159.14 & 126.43 & & (Ref. 31) \\
\hline $\mathrm{Ti}_{3} \mathrm{SiC}_{2}$ & 370.47 & 349.71 & 155.43 & 97.22 & 112.11 & 192.61 & 140.11 & 338.31 & \\
& 362.00 & 349.64 & 149.03 & 89.39 & 100.86 & 184.07 & 138.92 & & (Ref. 31) \\
\hline
\end{tabular}

Table 3: Elastic constants, bulk modulus (B), shear modulus (G), and Young's modulus (E)

B is bulk modulus, G is shear modulus, E is Young's modulus. Young's modulus E was obtained by the following formulas:

$$
E=\frac{9 B G}{3 B+G}
$$

Table 3 and Fig. 5 show the calculated elastic constants of $\mathrm{Ti}_{3} \mathrm{AlC}_{2}$ and $\mathrm{Ti}_{3} \mathrm{SiC}_{2}$. The hexagonal symmetry of the MAX phases result in anisotropic elastic constants $\left(\mathrm{C}_{11}\right.$ and $\mathrm{C}_{33}$ ). Both of the $\mathrm{Ti}_{3} \mathrm{AlC}_{2}$ and $\mathrm{Ti}_{3} \mathrm{SiC}_{2}$ are elastically stiffer along the a direction than along the c direction, and this agrees well with the calculated elastic constants reported by Wang et al [37], and the Materials Project [31]. The substitution of $\mathrm{Al}$ with $\mathrm{Si}$ highly increases $\mathrm{C}_{33}$ to a larger extent than $\mathrm{C}_{11}$, consistent with the fact that A-site substitution tends to affect in greater degree bonding along the $\mathrm{c}$ axis. The bulk and Young's modulus of $\mathrm{Ti}_{3} \mathrm{AlC}_{2}$ and $\mathrm{Ti}_{3} \mathrm{SiC}_{2}$ on finite-temperatures are compared in Fig. 6. The (low temperature) calculated bulk modulus agrees well with the experimented values:185 [34] and $187 \mathrm{GPa}$ [34] for $\mathrm{Ti}_{3} \mathrm{SiC}_{2}$ and 156 [38] and $165 \mathrm{GPa}$ [34] for $\mathrm{Ti}_{3} \mathrm{AlC}_{2}$. The calculated Young's modulus agrees well with the experimented values reported by Radovic et al [15], Wang et al [35] and Finkel et al [34]. The $\mathrm{Ti}_{3} \mathrm{SiC}_{2}$ has higher bulk modulus and Young's modulus than those of the $\mathrm{Ti}_{3} \mathrm{AlC}_{2}$.

Generally, as mentioned above, the elastic modulus and thermal expansion are inversely related [12]. 
However, the $\mathrm{Ti}_{3} \mathrm{SiC}_{2}$, which has higher elastic modulus than $\mathrm{Ti}_{3} \mathrm{AlC}_{2}$, softens faster than $\mathrm{Ti}_{3} \mathrm{AlC}_{2}$ with increasing temperature. The bulk modulus gap between $\mathrm{Ti}_{3} \mathrm{SiC}_{2}$ and $\mathrm{Ti}_{3} \mathrm{AlC}_{2}$ at ground state is $27.40 \mathrm{GPa}$, and at $1000(\mathrm{~K})$ is $25.03 \mathrm{GPa}$. The Young's modulus gap between the $\mathrm{Ti}_{3} \mathrm{SiC}_{2}$ and $\mathrm{Ti}_{3} \mathrm{AlC}_{2}$ at ground state is $34.22 \mathrm{GPa}$, and at $1000(\mathrm{~K})$ is $24.71 \mathrm{GPa}$. The Coefficient of Thermal Expansion $(\mathrm{CTE})$ of $\mathrm{Ti}_{3} \mathrm{AlC}_{2}$ and $\mathrm{Ti}_{3} \mathrm{SiC}_{2}$ are compared in Fig. $7(\mathrm{a})$. The CTE of $\mathrm{Ti}_{3} \mathrm{SiC}_{2}$ is higher than that of $\mathrm{Ti}_{3} \mathrm{AlC}_{2}$. The calculations for $\mathrm{Ti}_{3} \mathrm{SiC}_{2}$ overestimates the experimentally determined CTE by Zinkle [39].

The calculated thermal expansion of a and $\mathrm{c}$ lattice agrees well with experimented values reported by Scabarozi et al [40]. The calculated a lattice parameter of $\mathrm{Ti}_{3} \mathrm{SiC}_{2}$ below $\sim 650 \mathrm{~K}$ is lower than that of $\mathrm{Ti}_{3} \mathrm{AlC}_{2}$, however, the thermal expansion of the $\mathrm{Si}$ is higher than the $\mathrm{Al}$, and a lattice parameter of the $\mathrm{Ti}_{3} \mathrm{SiC}_{2}$ is higher than that of the $\mathrm{Ti}_{3} \mathrm{AlC}_{2}$ above $\sim 650 \mathrm{~K}$. Not surprisingly, there is a certain measure of anisotropy in the thermal expansion tensor. The thermal expansion of a lattice, $\alpha_{a}$, and c lattice, $\alpha_{c}$ of $\mathrm{Ti}_{3} \mathrm{SiC}_{2}$ are $9.1\left(10^{-6} \cdot \mathrm{K}^{-1}\right)$ and $11.0\left(10^{-6} \cdot \mathrm{K}^{-1}\right)$, respectively. The thermal expansion of $\alpha_{a}$ and $\alpha_{c}$ of $\mathrm{Ti}_{3} \mathrm{AlC}_{2}$ are $8.2\left(10^{-6} \cdot \mathrm{K}^{-1}\right)$ and $11.6\left(10^{-6} \cdot \mathrm{K}^{-1}\right)$. The A element mostly vibrates along a direction, which agrees well with the anisotropic atomic vibration of $\mathrm{A}$ element with highest frequency within basal plane reported by Lane et al [10]. The $\mathrm{Ti}_{3} \mathrm{AlC}_{2}$ compound is clearly more anisotropic (in terms of lattice thermal expansion) than $\mathrm{Ti}_{3} \mathrm{SiC}_{2}$, and this may be related to the different bond strengths when Si substitutes $\mathrm{Al}$.

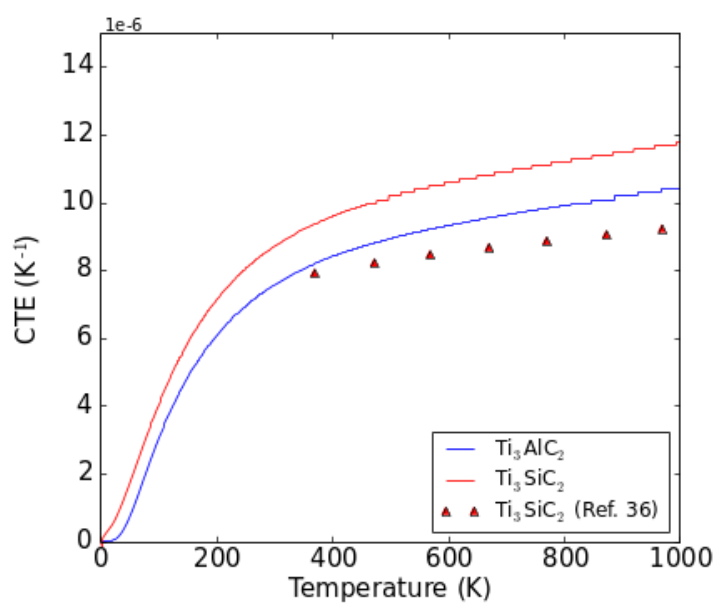

(a) Coefficient of Thermal Expansion (CTE)

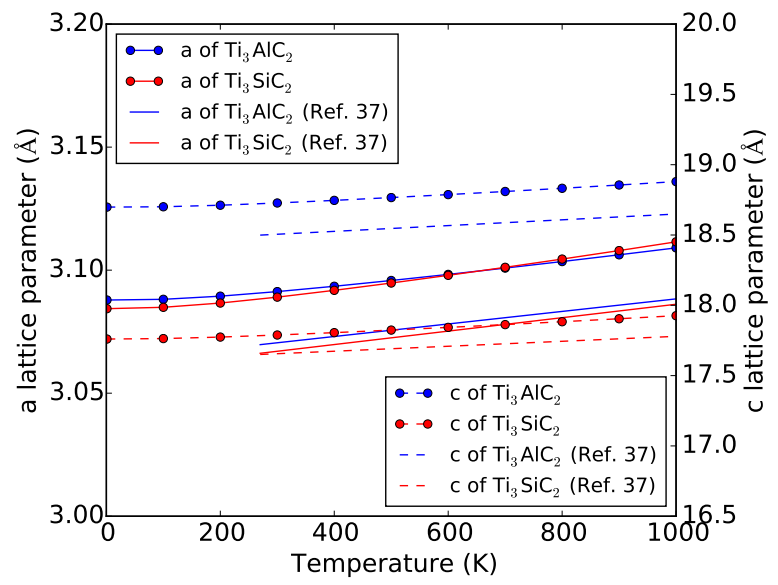

(b) Lattice parameter versus temperatures

Figure 7: Coefficient of Thermal Expansion (CTE) of the $\mathrm{Ti}_{3} \mathrm{SiC}_{2}$ and $\mathrm{Ti}_{3} \mathrm{AlC}_{2}$. The calculated $\mathrm{CTE}$ of $\mathrm{Ti}_{3} \mathrm{SiC}_{2}$ is compared to the experimental value of CTE reported by Zinkle [39]. The calculated lattice parameters versus temperatures are compared to the experimental value of lattice parameters reported by Scabarozi et al [40].

The total and partial phonon density of states of the are shown in Fig. 8 in good agreement with the calculated phonon density of states reported by Togo et al [11]. The partial phonon density of states were investigated to study thermal expansion of the $\mathrm{Ti}_{3} \mathrm{SiC}_{2}$ and $\mathrm{Ti}_{3} \mathrm{AlC}_{2}$. The phonon DOS of the $\mathrm{Ti}$ and $\mathrm{C}$ show similarity in both of the $\mathrm{Ti}_{3} \mathrm{SiC}_{2}$ and $\mathrm{Ti}_{3} \mathrm{AlC}_{2}$ phases. Both of the phonon DOS of the $\mathrm{Al}$ and $\mathrm{Si}$ have low frequencies wherein acoustic region (under $15 \mathrm{THz}$ ). However, the area under the curve of phonon DOS of $\mathrm{Si}$ and $\mathrm{Al}$ are, respectively, 6.40 and 3.89. The higher phonon DOS in acoustic region of the silicon than that of the aluminum agrees well with the calculated thermal expansion coefficient and calculated lattice parameters on finite-temperatures as shown in Fig. 8.

The thermodynamic properties of $\mathrm{Ti}_{3} \mathrm{AlC}_{2}$ and $\mathrm{Ti}_{3} \mathrm{SiC}_{2}$ are shown in Fig. 9. The thermodynamic calculation considers vibrational, electronic and anharmonic contributions. Below $250 \mathrm{~K}$, the calculated heat capacity of $\mathrm{Ti}_{3} \mathrm{SiC}_{2}$ agrees well with experimented values reported by Drulis et al [41]. Above $300 \mathrm{~K}$, the calculations slightly overestimate the heat capacity than experiments [3]. The heat capacity of both of the $\mathrm{Ti}_{3} \mathrm{AlC}_{2}$ and $\mathrm{Ti}_{3} \mathrm{SiC}_{2}$ are identical, which agrees well with other calculation reported by Ali et al [42]. The heat capacity was calculated from the free energy as a function of temperature as shown in Eq. 15. It 


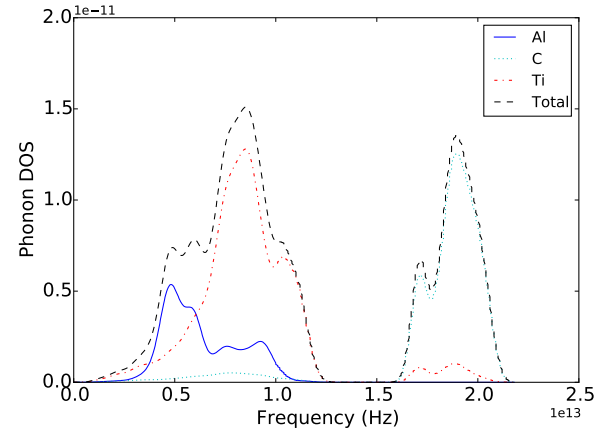

(a) $\mathrm{Ti}_{3} \mathrm{AlC}_{2}$

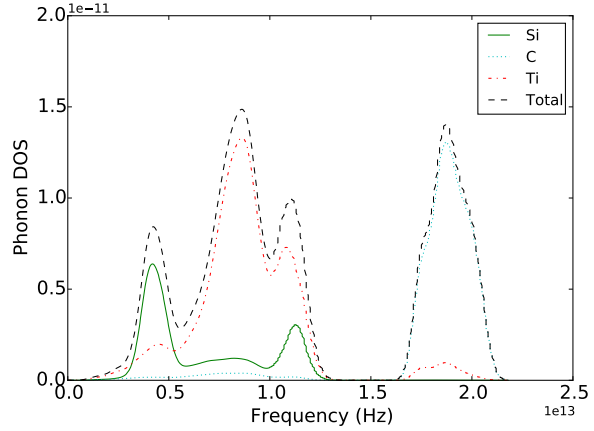

(b) $\mathrm{Ti}_{3} \mathrm{SiC}_{2}$

Figure 8: Total and partial Phonon Density of States (PDOS) of a) $\mathrm{Ti}_{3} \mathrm{AlC}_{2}$ and b) $\mathrm{Ti}_{3} \mathrm{SiC}_{2}$. The dashed, dashed-dotted and dotted curves denote total, Ti and $\mathrm{C}$, respectively, and the solid line denotes $\mathrm{Al}$ and $\mathrm{Si}$.

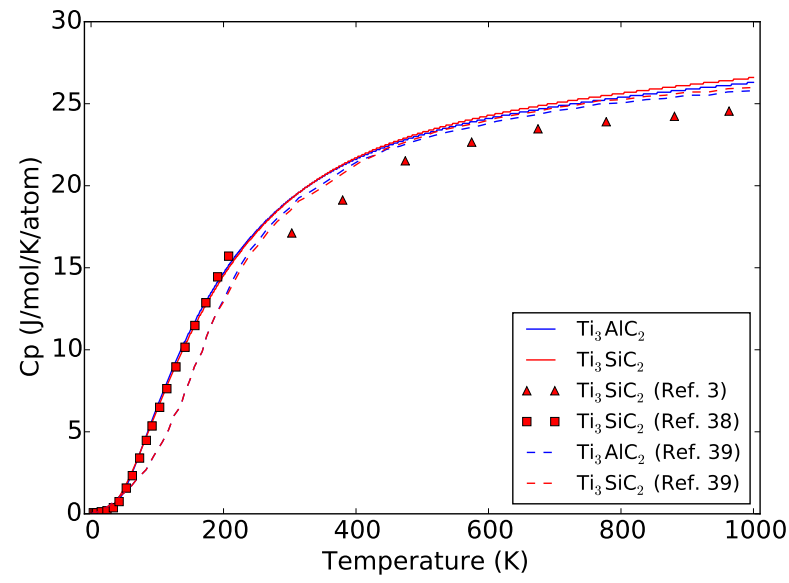

(a) Heat capacity, $\mathrm{C}_{p}$

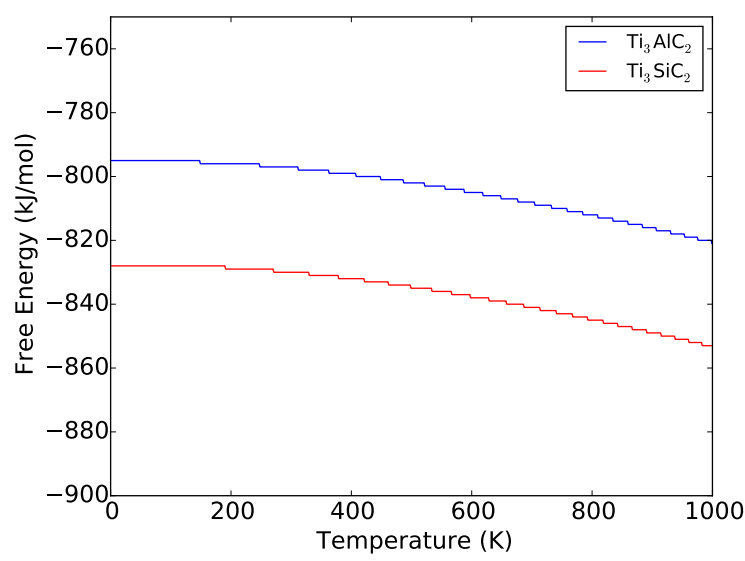

(b) Free energy, F

Figure 9: (a) Heat capacities and (b) free energies as a function of temperature. The red line and blue line denote $\mathrm{C}_{p}$ of $\mathrm{Ti}_{3} \mathrm{SiC}_{2}$ and $\mathrm{Ti}_{3} \mathrm{AlC}_{2}$, respectively. For the comparison, the square [3] and triangle [41] depict the experimental values of $\mathrm{C}_{p}$ of $\mathrm{Ti}_{3} \mathrm{SiC}_{2}$ below $250 \mathrm{~K}$ and above $300 \mathrm{~K}$, respectively. The calculated heat capacities are also compared to other computational results reported by Ali et al [42]. 
is resulted by the identical vibrational, electronic and anharmonic contributions of both of the $\mathrm{Ti}_{3} \mathrm{AlC}_{2}$ and $\mathrm{Ti}_{3} \mathrm{SiC}_{2}$ phases.

\section{Conclusions}

The structural, elastic and thermodynamic properties of $\mathrm{Ti}_{3} \mathrm{AlC}_{2}$ and $\mathrm{Ti}_{3} \mathrm{SiC}_{2}$ were investigated in this study. The calculated results of those properties of $\mathrm{Ti}_{3} \mathrm{AlC}_{2}$ and $\mathrm{Ti}_{3} \mathrm{SiC}_{2}$ agree well with the experimental data. The calculated results shows the anisotropic properties of $\mathrm{Ti}_{3} \mathrm{AlC}_{2}$ and $\mathrm{Ti}_{3} \mathrm{SiC}_{2}$. The substitution of $\mathrm{Al}$ with $\mathrm{Si}$ highly effect to the c directional properties, such as lattice parameter and elastic constants. The directly related elastic modulus and thermal expansion is investigated with the finite-temperatures elastic modulus and phonon density of states. The thermodynamic properties were calculated with the vibrational, electronic and anharmonic contributions. We investigated the thermodynamic properties (free energy, entropy and heat capacity) of $\mathrm{Ti}_{3} \mathrm{AlC}_{2}$ and $\mathrm{Ti}_{3} \mathrm{SiC}_{2}$ on finite-temperatures.

\section{Acknowledgments}

This work was supported by the grant NSF-DMR-1410983. AT acknowledges support from DMR0844082, while TD acknowledges support from DOE (through LLNL) under contract DE-AC52-07NA27344. First-principles calculations were carried out in the Texas A\&M Supercomputing Facility at Texas A\&M University.

\section{References}

[1] M. W. Barsoum, MAX phases: properties of machinable ternary carbides and nitrides, John Wiley \& Sons, 2013.

[2] M. Radovic, M. W. Barsoum, Max phases: Bridging the gap between metals and ceramics, American Ceramics Society Bulletin 92 (3) (2013) 20-27.

[3] M. W. Barsoum, The $\mathrm{m} n+1$ ax $\mathrm{n}$ phases: a new class of solids: thermodynamically stable nanolaminates, Progress in Solid State Chemistry 28 (1) (2000) 201-281.

[4] M. W. Barsoum, M. Radovic, Mechanical properties of the MAX phases, in: K. H. J. Buschow, R. Cahn, M. Flemings, B. Ilschner, E. Kramer, S. Mahajan, P. Veyssiere (Eds.), Encyclopedia of Materials: Science and Technology, Elsevier, Amsterdam, 2004, pp. 1-16.

[5] M. W. Barsoum, M. Radovic, Elastic and mechanical properties of the max phases, Annual review of materials research 41 (2011) 195-227.

[6] Z. Sun, Progress in research and development on MAX phases: A family of layered ternary compounds, International Materials Reviews 56 (3) (2011) 143-166.

[7] W. Jeitschko, H. Nowotny, Die kristallstruktur von ti3sic2ein neuer komplexcarbid-typ, Monatshefte für Chemie und verwandte Teile anderer Wissenschaften 98 (2) (1967) 329-337.

[8] M. A. Pietzka, J. C. Schuster, Phase equilibria in the quaternary system ti-al-c-n, Journal of the American Ceramic Society 79 (9) (1996) 2321-2330.

[9] M. W. Barsoum, T. El-Raghy, Synthesis and characterization of a remarkable ceramic: Ti3sic2, Journal of the American Ceramic Society 79 (7) (1996) 1953-1956.

[10] N. J. Lane, S. C. Vogel, M. W. Barsoum, High-temperature neutron diffraction and the temperaturedependent crystal structures of the $\mathrm{m}$ a x phases ti 3 sic 2 and ti 3 gec 2, Physical Review B 82 (17) (2010) 174109. 
[11] A. Togo, L. Chaput, I. Tanaka, G. Hug, First-principles phonon calculations of thermal expansion in ti 3 sic 2, ti 3 alc 2, and ti 3 gec 2, Physical Review B 81 (17) (2010) 174301.

[12] V. Milman, B. Winkler, M. Probert, Stiffness and thermal expansion of zrb2: an ab initio study, Journal of physics: Condensed matter 17 (13) (2005) 2233.

[13] T. Duong, S. Gibbons, R. Kinra, R. Arróyave, Ab-initio aprroach to the electronic, structural, elastic, and finite-temperature thermodynamic properties of ti2ax $(\mathrm{a}=\mathrm{al}$ or ga and $\mathrm{x}=\mathrm{c}$ or $\mathrm{n})$, Journal of Applied Physics 110 (9) (2011) 093504.

[14] T. C. Duong, N. Singh, R. Arróyave, First-principles calculations of finite-temperature elastic properties of ti 2 alx $(x=$ c or $n)$, Computational Materials Science 79 (2013) 296-302.

[15] M. Radovic, M. Barsoum, A. Ganguly, T. Zhen, P. Finkel, S. Kalidindi, E. Lara-Curzio, On the elastic properties and mechanical damping of ti 3 sic 2, ti 3 gec 2, ti 3 si 0.5 al 0.5 c 2 and ti 2 alc in the 300-1573k temperature range, Acta Materialia 54 (10) (2006) 2757-2767.

[16] S. Amini, M. W. Barsoum, T. El-Raghy, Synthesis and mechanical properties of fully dense ti2sc, Journal of the American Ceramic Society 90 (12) (2007) 3953-3958.

[17] S. Amini, A. Zhou, S. Gupta, A. DeVillier, P. Finkel, M. W. Barsoum, Synthesis and elastic and mechanical properties of cr 2 gec, Journal of Materials Research 23 (08) (2008) 2157-2165.

[18] W. Kohn, L. J. Sham, Self-consistent equations including exchange and correlation effects, Physical Review 140 (4A) (1965) A1133.

[19] G. Kresse, J. Furthmüller, Efficient iterative schemes for ab initio total-energy calculations using a plane-wave basis set, Phys. Rev. B 54 (1996) 11169-11186. doi:10.1103/PhysRevB.54.11169.

[20] G. Kresse, J. Furthmller, Efficiency of ab-initio total energy calculations for metals and semiconductors using a plane-wave basis set, Computational Materials Science 6 (1) (1996) 15 - 50. doi:http://dx.doi.org/10.1016/0927-0256(96)00008-0.

[21] A. D. Becke, A new mixing of hartree-fock and local density-functional theories, The Journal of Chemical Physics 98 (2) (1993) 1372-1377.

[22] J. P. Perdew, J. Chevary, S. Vosko, K. A. Jackson, M. R. Pederson, D. Singh, C. Fiolhais, Atoms, molecules, solids, and surfaces: Applications of the generalized gradient approximation for exchange and correlation, Physical Review B 46 (11) (1992) 6671.

[23] M. Methfessel, A. T. Paxton, High-precision sampling for brillouin-zone integration in metals, Phys. Rev. B 40 (1989) 3616-3621. doi:10.1103/PhysRevB.40.3616.

[24] Y. Wang, J. Wang, H. Zhang, V. Manga, S. Shang, L. Chen, Z. Liu, A first-principles approach to finite temperature elastic constants, Journal of Physics: Condensed Matter 22 (22) (2010) 225404.

[25] A. Van de Walle, M. Asta, G. Ceder, The alloy theoretic automated toolkit: A user guide, Calphad 26 (4) (2002) 539-553.

[26] S. Wei, M. Chou, Ab initio calculation of force constants and full phonon dispersions, Physical review letters 69 (19) (1992) 2799.

[27] A. Van De Walle, G. Ceder, The effect of lattice vibrations on substitutional alloy thermodynamics, Reviews of Modern Physics 74 (1) (2002) 11.

[28] J. C. Slater, A simplification of the hartree-fock method, Physical Review 81 (3) (1951) 385. 
[29] M. Naguib, M. Kurtoglu, V. Presser, J. Lu, J. Niu, M. Heon, L. Hultman, Y. Gogotsi, M. W. Barsoum, Two-dimensional nanocrystals produced by exfoliation of ti3alc2, Advanced Materials 23 (37) (2011) $4248-4253$.

[30] E. Kisi, J. Crossley, S. Myhra, M. Barsoum, Structure and crystal chemistry of ti 3 sic 2, Journal of Physics and Chemistry of Solids 59 (9) (1998) 1437-1443.

[31] A. Jain, S. P. Ong, G. Hautier, W. Chen, W. D. Richards, S. Dacek, S. Cholia, D. Gunter, D. Skinner, G. Ceder, et al., Commentary: The materials project: A materials genome approach to accelerating materials innovation, Apl Materials 1 (1) (2013) 011002.

[32] A. Savin, R. Nesper, S. Wengert, T. F. Fässler, Elf: The electron localization function, Angewandte Chemie International Edition in English 36 (17) (1997) 1808-1832.

[33] Y. Zhou, Z. Sun, X. Wang, S. Chen, Ab initio geometry optimization and ground state properties of layered ternary carbides ti3mc2 $(\mathrm{m}=\mathrm{al}$, si and ge), Journal of Physics: Condensed Matter 13 (44) (2001) 10001.

[34] P. Finkel, M. Barsoum, T. El-Raghy, Low temperature dependencies of the elastic properties of ti4aln3, ti3al1. 1c1. 8, and ti3sic2, Journal of Applied physics 87 (2000) 1701-1703.

[35] J. Wang, Y. Zhou, Recent progress in theoretical prediction, preparation, and characterization of layered ternary transition-metal carbides, Annual Review of Materials Research 39 (2009) 415-443.

[36] R. Hill, The elastic behaviour of a crystalline aggregate, Proceedings of the Physical Society. Section A 65 (5) (1952) 349.

[37] J.-Y. Wang, Y.-C. Zhou, Polymorphism of ti 3 sic 2 ceramic: first-principles investigations, Physical Review B 69 (14) (2004) 144108.

[38] H. Zhang, X. Wu, K. G. Nickel, J. Chen, V. Presser, High-pressure powder x-ray diffraction experiments and ab initio calculation of ti3alc2, Journal of Applied Physics 106 (1) (2009) 013519.

[39] S. Zinkle, 7.3 physical and thermal mechanical characterization of non-irradiated max phase.

[40] T. Scabarozi, S. Amini, O. Leaffer, A. Ganguly, S. Gupta, W. Tambussi, S. Clipper, J. Spanier, M. Barsoum, J. Hettinger, et al., Thermal expansion of select $\mathrm{m} \mathrm{n}$ ax $\mathrm{n} \mathrm{m}=$ early transition metal, $\mathrm{a}=$ a group element, $\mathrm{x}=\mathrm{c}$ or $\mathrm{n}$ phases measured by high temperature $\mathrm{x}$-ray diffraction and dilatometry, Journal of Applied Physics 105 (2009) 013543.

[41] M. K. Drulis, A. Czopnik, H. Drulis, M. Barsoum, Low temperature heat capacity and magnetic susceptibility of ti3sic2, Journal of applied physics 95 (2004) 128-133.

[42] M. Ali, A. Islam, M. Hossain, F. Parvin, Phase stability, elastic, electronic, thermal and optical properties of ti 3 al 1- x si x c 2 (0 x 1): First principle study, Physica B: Condensed Matter 407 (21) (2012) $4221-4228$. 

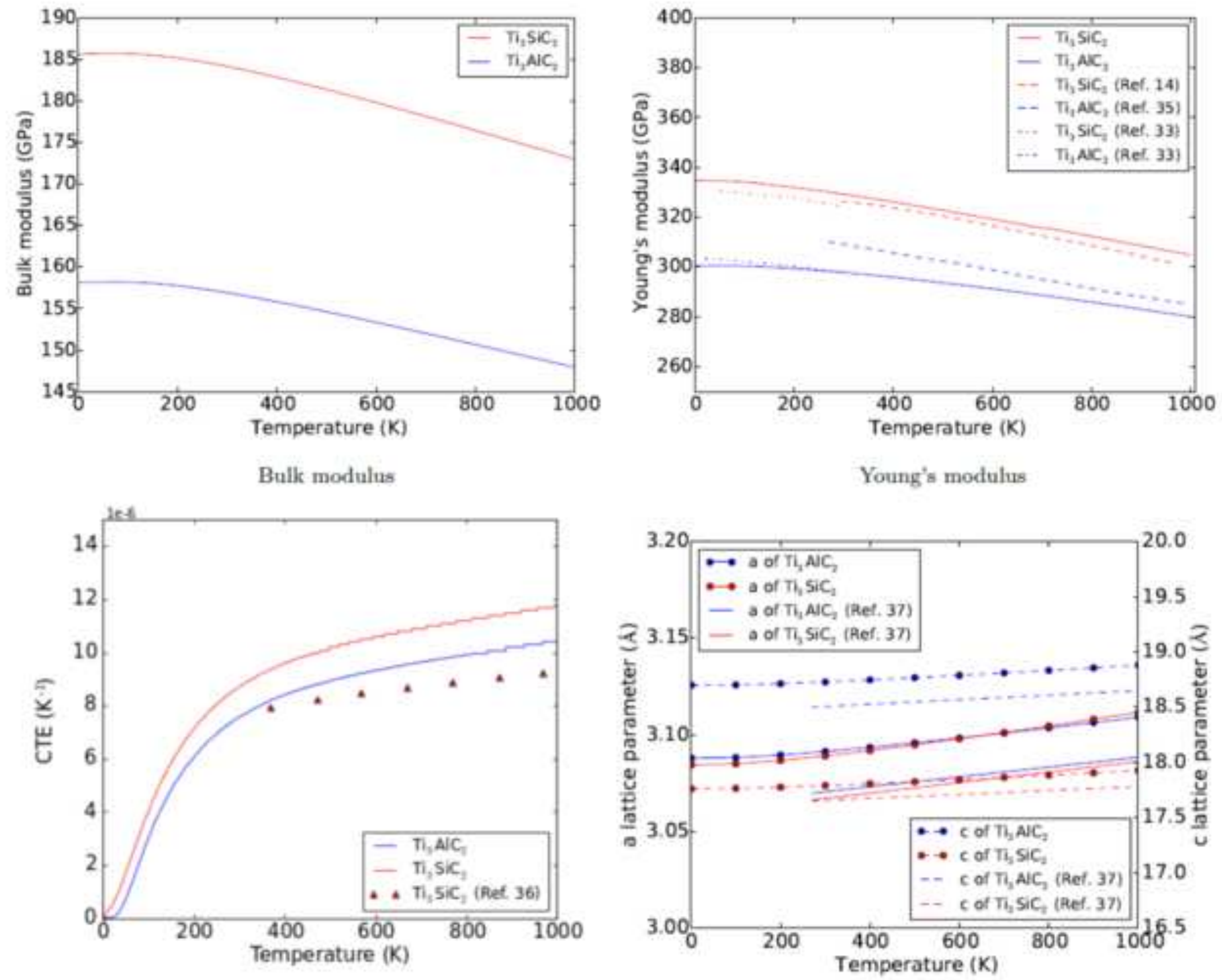

Coefficient of Thermal Expansion (CTE)

Lattice parameter versus temperatures 